\title{
Evaluation of the hybrid-dynamic conformal arc therapy technique for radiotherapy of lung cancer
}

\author{
Sung Joon Kim, $\mathrm{MS}^{1 *}$, Jeong Won Lee, MD, PhD ${ }^{*}$, Min Kyu Kang, MD², Jae-Chul Kim, MD, PhD², \\ Jeong Eun Lee, MD, PhD², Shin-Hyung Park, MD, PhD², Mi Young Kim, MD³ Seoung-Jun Lee, PhD², \\ Soo-Ho Moon, $\mathrm{MS}^{3}$, Byoung-Soo Ko, MS \\ 'Department of Radiation Oncology, Kyungpook National University Chilgok Hospital, Daegu; \\ ${ }^{2}$ Department of Radiation Oncology, School of Medicine, Kyungpook National University, Daegu; \\ ${ }^{3}$ Department of Radiation Oncology, Kyungpook National University Hospital, Daegu, Korea
}

Purpose: A hybrid-dynamic conformal arc therapy (HDCAT) technique consisting of a single half-rotated dynamic conformal arc beam and static field-in-field beams in two directions was designed and evaluated in terms of dosimetric benefits for radiotherapy of lung cancer.

Materials and Methods: This planning study was performed in 20 lung cancer cases treated with the VERO system (BrainLAB $A G$, Feldkirchen, Germany). Dosimetric parameters of HDCAT plans were compared with those of three-dimensional conformal radiotherapy (3D-CRT) plans in terms of target volume coverage, dose conformity, and sparing of organs at risk.

Results: HDCAT showed better dose conformity compared with 3D-CRT (conformity index: $0.74 \pm 0.06$ vs. $0.62 \pm 0.06, p<0.001$ ). HDCAT significantly reduced the lung volume receiving more than $20 \mathrm{~Gy}\left(\mathrm{~V}_{20}: 21.4 \% \pm 8.2 \%\right.$ vs. $24.5 \% \pm 8.8 \%, \mathrm{p}<0.001 ; \mathrm{V}_{30}: 14.2 \%$ $\pm 6.1 \%$ vs. $15.1 \% \pm 6.4 \%, p=0.02 ; V_{40}: 8.8 \% \pm 3.9 \%$ vs. $10.3 \% \pm 4.5 \%, p<0.001 ;$ and $V_{50}: 5.7 \% \pm 2.7 \%$ vs. $\left.7.1 \% \pm 3.2 \%, p<0.001\right)$, $V_{40}$ and $V_{50}$ of the heart $\left(V_{40}: 5.2 \pm 3.9 \mathrm{~Gy}\right.$ vs. $7.6 \pm 5.5 \mathrm{~Gy}, \mathrm{p}<0.001 ; \mathrm{V}_{50}: 1.8 \pm 1.6 \mathrm{~Gy}$ vs. $\left.3.1 \pm 2.8 \mathrm{~Gy}, \mathrm{p}=0.001\right)$, and the maximum spinal cord dose $(34.8 \pm 9.4$ Gy vs. $42.5 \pm 7.8 \mathrm{~Gy}, \mathrm{p}<0.001)$ compared with 3D-CRT.

Conclusions: HDCAT could achieve highly conformal target coverage and reduce the doses to critical organs such as the lung, heart, and spinal cord compared to 3D-CRT for the treatment of lung cancer patients.

Keywords: Lung neoplasms, Radiotherapy planning, Three-dimensional conformal radiotherapy, Arc therapy

\section{Introduction}

Radiotherapy (RT) alone or with chemotherapy is an important treatment for locally advanced lung cancer. Although a radiation dose higher than $60 \mathrm{~Gy}$ is important for better tumor control [1], the radiation dose is restricted by the tolerance of organs at risk (OARs), including the lungs, spinal cord, heart, and esophagus. Therefore, an effective delivery of the radiation dose to the target is required while preserving the normal tissues. After the introduction of three-dimensional conformal radiotherapy (3D-CRT), RT-related toxicities have decreased $[2,3]$.

Recently, intensity-modulated radiotherapy (IMRT) is being used for the treatment of lung cancer because it can improve

Received 30 March 2018, Revised 16 July 2018, Accepted 16 August 2018.

Correspondence: Min Kyu Kang, MD, Department of Radiation Oncology, Kyungpook National University Chilgok Hospital, 807 Hoguk-ro, Buk-gu, Daegu 41404, Korea. Tel: +82-53-200-2653, Fax: +82-53-200-2029, E-mail: mkkang@knu.ac.kr

*Both authors contributed equally to this work.

(c) This is an Open Access article distributed under the terms of the Creative Commons Attribution Non-Commercial License (http://creativecommons.org/ licenses/by-nc/4.0/) which permits unrestricted non-commercial use, distribution, and reproduction in any medium, provided the original work is properly cited.

www.e-roj.org 
dose conformity and lower radiation doses to OARs compared with 3D-CRT [4-7]. Even though IMRT has some issues related to the dose uncertainty for moving tumors and a complex process of treatment planning and quality assurance $[4,8]$, clinical evidence supports the use of IMRT for lung cancer [9]. Even though IMRT can be useful in some patients with a big or complex target volume, some others can be effectively treated with 3D-CRT.

Therefore, we designed a new technique, hybrid-dynamic conformal arc radiotherapy (HDCAT), to improve the quality of 3D-CRT. HDCAT is a type of conformal arc RT technique that delivers a single arc with static conformal fields. In this study, we evaluated the dosimetric advantages of HDCAT over 3D-CRT for the treatment of lung cancer.

\section{Materials and Methods}

The Institutional Review Board of Kyungpook National University Chilgok Hospital approved this study and waived the requirement for informed patient consent (No. 2016-12026).

\section{Patient selection}

Twenty consecutive patients who were treated with HDCAT for lung cancer using the VERO system (BrainLAB AG, Feldkirchen, Germany) from November 2015 to March 2016 were retrospectively enrolled for this planning study. According to the discretion of physicians in this institution, patients with central tumors or tumors close to or invading into the mediastinum were treated with HDCAT unless the target volume exceeded the field size of the VERO system (maximum, $15 \mathrm{~cm} \times 15 \mathrm{~cm}$ ). Fourteen patients had tumors abutting on or invading into large bronchi with or without regional lymph node metastasis. Four patients had tumors close to or invading into the mediastinum. One patient had subcarinal lymph node recurrence, and one patient had left hilar lymph node recurrence. Nineteen patients had non-small cell lung cancer and one had small cell lung cancer. All patients had locally advanced disease (c/rT1-4NO-2) in the thorax based on the 7th edition of the American Joint Committee on Cancer staging system [10], and three of them had distant metastasis. The study included 19 men and 1 woman, with a median age of 72 years (range, 51 to 81 years).

\section{Simulation and target volume delineation}

Patients lay in a supine position with both arms raised and were immobilized with a wing board and a vacuum bag. All patients underwent four-dimensional computed tomography (4D-CT) scans with slices of 3-mm thickness from the mandible to the mid-abdomen to include the whole lung using a Brilliance CT Big Bore (Philips, Amsterdam, the Netherlands). During 4D-CT scanning, the respiration of the patient was monitored with a Real-time Positioning Management (RPM) respiratory gating system (Varian Medical Systems, Palo Alto, CA, USA). The gross tumor volume (GTV) was defined as the primary tumor and involved regional lymph nodes. To generate the internal target volume (ITV), GTVs were manually delineated while reviewing all 10 respiratory phases of 4D-CT and expanded with a 6-mm margin considering the anatomical boundaries. The planning target volume (PTV) was generated by adding a 5-mm margin to the ITV. The target volumes and normal organs, including the total lung, heart, and spinal cord, were delineated on the untagged image set of 4D-CT images, which is a time weighted reconstruction with a true Hounsfield unit representation.

\section{Treatment planning}

HDCAT plans used for the treatment of patients were not modified for this study except the dose prescription for dosimetric comparisons and 3D-CRT plans were generated with an effort to minimize the doses to OARs as much as possible. All plans were generated considering the recommended normal tissue dose-volume constraints [11]. Plans were generated with iPlan RT v4.5 (BrainLAB AG) for the VERO system with a 6-MV photon beam. Dose distribution was calculated with $X$-ray voxel Monte Carlo (XVMC) algorithm with a spatial resolution of $2 \mathrm{~mm}$ and mean variance of $2 \%$.

The prescribed dose to the PTV was 66 Gy in 33 fractions. The planning objective was to achieve a minimum dose to ITV greater than 98\% prescribed dose. The HDCAT plan consisted of a single dynamic conformal arc (DCA) rotating $180^{\circ}$ and static conformal fields with the same isocenter (Fig. 1). The starting point of the DCA was chosen to minimize the directly irradiated lung volume. Static conformal fields were arranged in the two directions, namely the starting and ending point of the DCA, in order to make the skewed dose distribution caused by DAC homogenous. Static conformal beams in one direction consisted of 3-4 field-in-field beams for the control of dose distribution and dose of OARs. A set of field-in-field beams was designed to mimic the motion of dynamic wedge in simple cases. Or multileaf collimators (MLCs) were manually manipulated to shield the areas receiving higher dose. Beam weight of DCA and static conformal beam groups was adjusted to make the dose distribution homogeneous and beam weight 
of DCA was $79.1 \% \pm 10.6 \%$. The 3D-CRT plan consisted of anterior-posterior, posterior-anterior, left-anterior-oblique or right-anterior-oblique, left-posterior-oblique or rightposterior-oblique fields, and an optional additional field to reduce the dose to the spinal cord, whose arrangement was similar to the shape of a $\mathrm{K}$ or an inverse $\mathrm{K}$. MLC margins were $3 \mathrm{~mm}$ for HDCAT plan and $5 \mathrm{~mm}$ for 3D-CRT plan, respectively.

\section{Dosimetric evaluation and statistical analysis}

For the comparison of target dose coverage between HDCAT and 3D-CRT plans, he conformity index $(\mathrm{CI})$ used by MacFarlane et al. [12] and homogeneity index (HI) used by Jiang et al. [13] were calculated. The $\mathrm{Cl}$ was defined as

$$
\mathrm{Cl}=(\text { Cover factor }) \times(\text { Spill factor })=\frac{\mathrm{V}_{95 \mathrm{PTV}}}{\mathrm{V}_{\mathrm{PTV}}} \times \frac{\mathrm{V}_{95 \mathrm{PTV}}}{\mathrm{V}_{\text {95body }}}
$$

where $V_{95 \text { PTV }}$ and $V_{\text {95body }}$ are the volumes of the PTV and body, respectively, receiving at least $95 \%$ of the prescription dose, and $V_{\text {PTV }}$ is the volume of the PTV. The better the dose conformity is, the closer the $\mathrm{Cl}$ value approaches 1 . The $\mathrm{HI}$ was defined as

$$
H I=\frac{D_{5 P T V}}{D_{95 P T V}}
$$

where $D_{5 P T V}$ and $D_{95 P T V}$ correspond to the dose delivered to $5 \%$ and 95\% of the PTV volume, respectively. The lower (closer to 1) $\mathrm{HI}$ is, the better the dose homogeneity.

Parameters for OARs were acquired from the dose-volume histograms (DVHs) of the HDCAT and 3D-CRT plans [14-17]; $V_{5 / 10 / 13 / 20 / 30 / 40 / 50}$ (the percentage volumes receiving 5, 10, 13, 20, 30, 40, and $50 \mathrm{~Gy}$, respectively) and Dmean (mean dose) of the total lung, $V_{40}$ and $V_{50}$ (the percentage volumes receiving 40 and $50 \mathrm{~Gy}$, respectively) of the heart, and Dmax (maximum dose) of the spinal cord. The volume of the total lung was defined as the volume of both lung minus GTV. $V_{5}$ and $V_{50}$ of the total lung, $V_{50}$ of the heart, and Dmax of the spinal cord were analyzed using the Wilcoxon signed-rank test. The rest dosimetric parameters were analyzed using the paired t-test. A $p$-value of $<0.05$ was considered statistically significant.

\section{Results}

The median volume of the PTV was $214.94 \mathrm{~mL}$ (range, 82.63 to $467.13 \mathrm{~mL}$ ). The target coverage parameters of HDCAT and 3D-CRT are summarized in Table 1. HDCAT provided better conformity compared with 3D-CRT (mean Cl: 0.74 vs. 0.62; $p<0.001)$ but they showed similar homogeneity of target

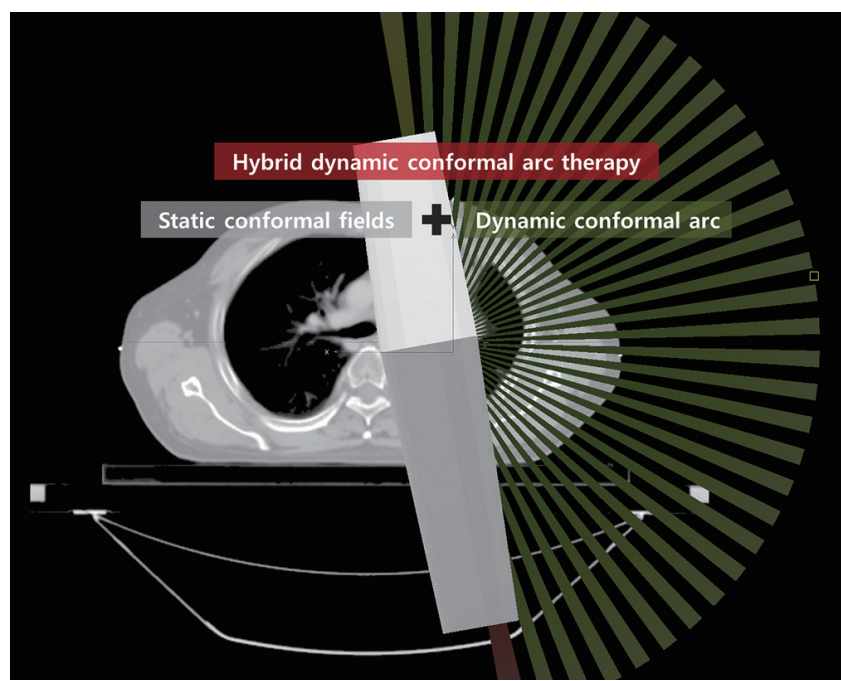

Fig. 1. Hybrid-dynamic conformal arc radiotherapy plan, consisting of a single dynamic conformal arc (dark green) and static conformal fields (gray).

Table 1. Comparison of the target dose-volume parameters for HDCAT and 3D-CRT

\begin{tabular}{cccc}
\hline & HDCAT & 3D-CRT & p-value \\
\hline $\mathrm{Cl}$ & $0.74 \pm 0.06$ & $0.62 \pm 0.06$ & $<0.001^{\mathrm{a}}$ \\
$\mathrm{HI}$ & $1.10 \pm 0.02$ & $1.10 \pm 0.02$ & $0.616^{\mathrm{a}}$ \\
\hline
\end{tabular}

Values are presented as mean \pm standard deviation.

HDCAT, hybrid dynamic conformal arc radiotherapy; 3D-CRT, three-dimensional conformal radiotherapy; $\mathrm{Cl}$, conformity index $\mathrm{HI}$, homogeneity index.

${ }^{\text {a) }}$ Paired t-test.

coverage (mean HI: 1.10 vs. 1.10; $p=0.616$ ). Dose distributions and DVHs of an example case are shown in Figs. 2 and 3

Dosimetric parameters of OARs are shown in Table 2. The total lung volume receiving 20 to 50 Gy was significantly smaller in the HDCAT plan than in the 3D-CRT plan. The mean lung dose (MLD) tended to be smaller in the HDCAT plan (12.0 $\pm 3.6 \mathrm{~Gy}$ vs. $12.2 \pm 3.8 \mathrm{~Gy}, \mathrm{p}=0.065) . \mathrm{V}_{10}$ and $\mathrm{V}_{13}$ of the total lung were not different between the two plans. $V_{5}$ of the total lung was significantly larger for HDCAT $(50.8 \% \pm 14.7 \%$ vs. $41.6 \pm 13.0 \%, p<0.001)$. In addition, $V_{40}$ and $V_{50}$ of the heart and Dmax of the spinal cord were significantly lower in HDCAT compared with 3D-CRT.

\section{Discussion and Conclusion}

This planning study confirmed that HDCAT had dosimetric 

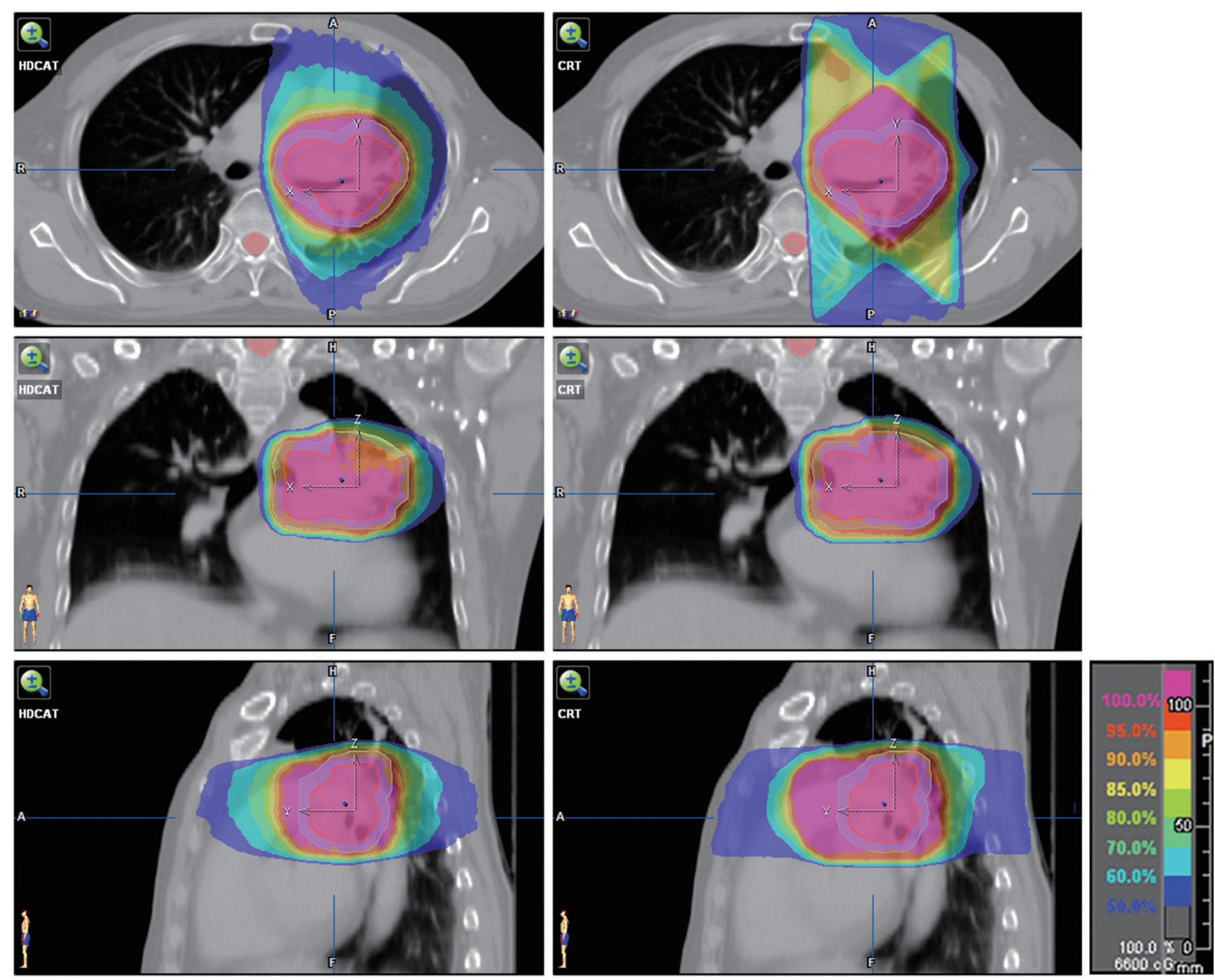

Fig. 2. Dose distributions of hybrid-dynamic conformal arc radiotherapy plan (left) and three-dimensional conformal radiotherapy plan (right). Internal target volume in red and planning target volume in cyan.

advantages over 3D-CRT in terms of the conformity and the doses to OARs.

Several studies reported that IMRT or volumetric modulated arc therapy (VMAT) could enhance $\mathrm{Cl}$ when they used the ratio of the volume of normal tissue and target receiving the prescribed dose for the calculation of $\mathrm{Cl}[5,18]$. Unlike these studies, we used a different formula for $\mathrm{Cl}$ that included the spill factor, which reflects a 'fall-off' in dose distribution, and the formula used in the current study is similar to that used by Jiang et al. [13]. Jiang et al. [13] reported that the mean values of $\mathrm{Cl}$ of IMRT and single-arc/partial-arc VMAT were 0.62 to 0.68 for locally advanced lung cancer. In the current study, the $\mathrm{Cl}$ of HDCAT $(0.74 \pm 0.06)$ was significantly better than that of 3D-CRT and seems not to be inferior to the results of IMRT and
VMAT [13]. Dose homogeneity of HDCAT was similar to that of IMRT and VMAT of other studies using the same formula for $\mathrm{HI}$ $[5,13]$.

The $V_{20}$ of the total lung and MLD are well-known parameters to estimate the probability of radiation pneumonitis $[19,20]$. The $V_{20}$ of the total lung for HDCAT was significantly less compared with 3D-CRT $(p<0.001)$, while MLD was not different between the two plans. Kristensen et al. [21] reported that MLD and $V_{10}$ were critical factors for fatal lung toxicity. There was no significant difference between the $V_{10}$ and $V_{13}$ for HDCAT and that of 3D-CRT in our study ( $p$ $=0.819$ and $p=0.097$, respectively). HDCAT had dosimetric benefits in the high dose ( $\geq 30 \mathrm{~Gy}$ ) irradiated area compared with 3D-CRT. Because the risk of lung fibrosis can increase 
Table 2. Comparison of doses to organs at risk

\begin{tabular}{|c|c|c|c|}
\hline & HDCAT & 3D-CRT & $p$-value \\
\hline \multicolumn{4}{|l|}{ Total lung } \\
\hline Mean dose (Gy) & $12.0 \pm 3.6$ & $12.2 \pm 3.7$ & $0.102^{a)}$ \\
\hline$V_{5}(\%)$ & $50.8 \pm 14.7$ & $41.6 \pm 13.0$ & $<0.001^{b)}$ \\
\hline$V_{10}(\%)$ & $32.5 \pm 11.9$ & $32.3 \pm 10.6$ & $0.819^{a)}$ \\
\hline$V_{13}(\%)$ & $26.9 \pm 10.2$ & $28.5 \pm 10.0$ & $0.097^{\mathrm{a})}$ \\
\hline$V_{20}(\%)$ & $21.4 \pm 8.2$ & $24.5 \pm 8.8$ & $<0.001^{\mathrm{a})}$ \\
\hline$V_{30}(\%)$ & $14.2 \pm 6.1$ & $15.1 \pm 6.4$ & $0.022^{\mathrm{a})}$ \\
\hline$V_{40}(\%)$ & $8.8 \pm 3.9$ & $10.3 \pm 4.5$ & $<0.001^{\text {a) }}$ \\
\hline$V_{50}(\%)$ & $5.7 \pm 2.7$ & $7.1 \pm 3.2$ & $<0.001^{\mathrm{b})}$ \\
\hline \multicolumn{4}{|l|}{ Heart } \\
\hline$V_{40}(\%)$ & $5.2 \pm 3.9$ & $7.6 \pm 5.5$ & $<0.001^{\mathrm{a})}$ \\
\hline$V_{50}(\%)$ & $1.8 \pm 1.6$ & $3.1 \pm 2.8$ & $0.001^{b)}$ \\
\hline \multicolumn{4}{|l|}{ Spinal cord } \\
\hline Dmax (Gy) & $34.8 \pm 9.4$ & $42.5 \pm 7.8$ & $<0.001^{b)}$ \\
\hline
\end{tabular}

Values are presented as mean \pm standard deviation.

HDCAT, hybrid dynamic conformal arc radiotherapy; 3D-CRT, three-dimensional conformal radiotherapy; Dmax, maximum dose.

${ }^{\text {a) }}$ Paired t-test. ${ }^{\text {b) }}$ Wilcoxon signed-rank test.

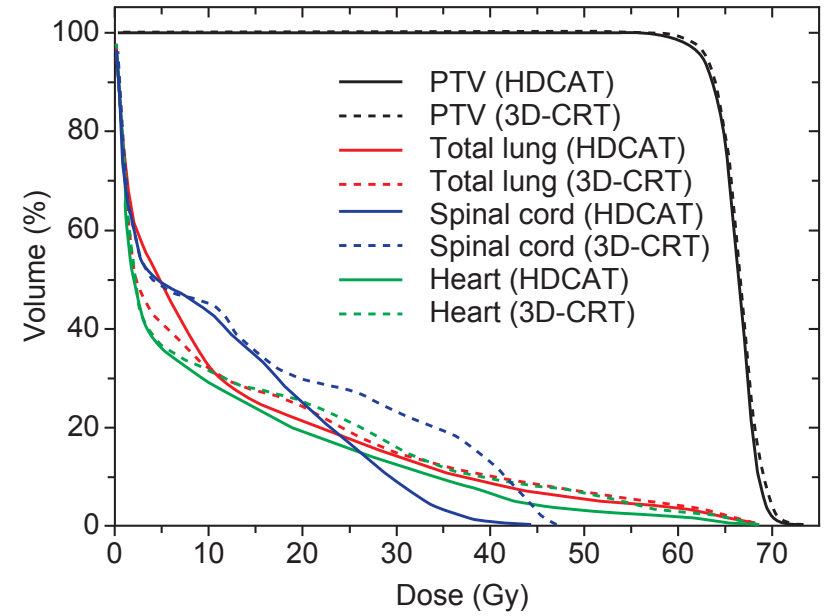

Fig. 3. Average dose-volume histograms of hybrid-dynamic conformal arc radiotherapy (HDCAT, solid line) and threedimensional conformal radiotherapy (3D-CRT, dashed line) plans.

with a radiation dose greater than $30 \mathrm{~Gy}[2,22]$, HDCAT could reduce the risk of lung fibrosis. Similar to the studies of IMRT $[5,18]$, the $V_{5}$ of the total lung for HDCAT was significantly higher compared with $3 \mathrm{D}-\mathrm{CRT}(50.8 \% \pm 14.7 \%$ vs. $41.6 \% \pm$ $13.0 \%, p<0.001)$. Even though some authors reported that the $V_{5}$ of the lung was a predictor for radiation pneumonitis after RT $[16,23]$, the association between low-dose irradiated volume and lung toxicity is controversial and there is no consensus on the threshold doses [4]. Nonetheless, the $V_{5}$ of the total lung for HDCAT was within the recommended dose of less than 65\% [11]. Therefore, the current study confirmed the dosimetric superiority of HDCAT compared with 3D-CRT in terms of radiation pneumonitis and fibrosis.

Cardiac complications can occur in 6\%-29\% of lung cancer patients [24-26]. Because high-dose irradiation to the heart can increase radiation-induced cardiac complications [14], the use of HDCAT could reduce the potential risk of cardiac toxicities. $V_{40}$ and $V_{50}$ of the heart was lower in HDCAT compared with 3D-CRT in the current study (Table 2), which might be attributed to the better conformity of HDCAT.

HDCAT could reduce the Dmax of the spinal cord in comparison to 3D-CRT. Because the angles of the beams for 3D-CRT are limited for the treatment of lung cancer as shown in Fig. 2, sometimes it is very difficult for a 3D-CRT plan to reduce the dose to the spinal cord because of the threedimensional relationship between the target and spinal cord. However, based on our experience, HDCAT could more easily reduce the dose to the spinal cord below the tolerance dose compared with 3D-CRT.

As HDCAT combining arc and conformal beams, other techniques using more than one type of beam have been reported. Chan et al. [18] used a Hybrid-RapidArc (H-RA) technique for lung cancer, which consists of two volumetricmodulated arcs and two static fields. Their study proved the superiority of H-RA over 3D-CRT and VMAT in dosimetric aspects of the lung and spinal cord. Sasaoka et al. [27] compared a combination of conformal dynamic-arc and five- 
static fields to other 3D-CRT techniques in whole pelvic RT for prostate cancer. The authors reported the best dosimetric outcomes in the rectum were achieved with a combination of conformal dynamic-arc and five-static fields, where the static fields were manually designed to shield the rectum included in the PTV. Static conformal fields in the two directions of HDCAT were manually designed to control dose distribution with multiple field-in-field beams, which can be simply replaced with conformal beams with a wedge compensator in other treatment machines, unlike VERO. Based on this experience, we currently apply the HDCAT technique with various arc angles to other locations of the body to reduce doses to the critical organs that are located close to the target.

There are some limitations in the current study, because this study was performed only with patients treated with VERO. The physical properties of VERO are slightly different from other treatment machines. Because of the maximum field size of 15 $\mathrm{cm} \times 15 \mathrm{~cm}$, we only treated patients with a relatively small tumor volume. Therefore, our results should be cautiously interpreted, keeping in mind that the volume of PTV in our study ranged from $82.6 \mathrm{~mL}$ to $467.1 \mathrm{~mL}$ (median, $214.9 \mathrm{~mL}$ ). In this volume range, we could not see a trend in the absolute value of the difference in all dosimetric parameters between HDCAT and 3D-CRT according to the PTV volume. For patients with a larger target volume to be treated, further studies comparing HDCAT with other treatment techniques, including 3D-CRT, IMRT, and VMAT, are warranted.

In conclusion, the HDCAT technique using a single halfrotated conformal arc and static field-in-field beams showed better target coverage and less doses to OARs such as the lung, heart, and spinal cord compared with 3D-CRT. Therefore, HDCAT is expected to reduce radiation-induced toxicities for lung cancer.

\section{Conflict of Interest}

No potential conflict of interest relevant to this article was reported.

\section{References}

1. Perez CA, Bauer M, Edelstein S, Gillespie BW, Birch R. Impact of tumor control on survival in carcinoma of the lung treated with irradiation. Int J Radiat Oncol Biol Phys 1986;12:539-47.

2. Kong FM, Ten Haken R, Eisbruch A, Lawrence TS. Non-small cell lung cancer therapy-related pulmonary toxicity: an update on radiation pneumonitis and fibrosis. Semin Oncol 2005;32(2
Suppl 3):S42-54

3. McGibney C, Holmberg O, McClean B, et al. Dose escalation of chart in non-small cell lung cancer: is three-dimensional conformal radiation therapy really necessary? Int J Radiat Oncol Biol Phys 1999;45:339-50.

4. Chan C, Lang S, Rowbottom C, Guckenberger M, Faivre-Finn C; IASLC Advanced Radiation Technology Committee. Intensitymodulated radiotherapy for lung cancer: current status and future developments. J Thorac Oncol 2014;9:1598-608.

5. Liu HH, Wang $X$, Dong $L$, et al. Feasibility of sparing lung and other thoracic structures with intensity-modulated radiotherapy for non-small-cell lung cancer. Int J Radiat Oncol Biol Phys 2004;58:1268-79.

6. Scorsetti M, Navarria P, Mancosu P, et al. Large volume unresectable locally advanced non-small cell lung cancer: acute toxicity and initial outcome results with rapid arc. Radiat Oncol 2010;5:94.

7. Sura S, Gupta V, Yorke E, Jackson A, Amols H, Rosenzweig KE. Intensity-modulated radiation therapy (IMRT) for inoperable non-small cell lung cancer: the Memorial SloanKettering Cancer Center (MSKCC) experience. Radiother Oncol 2008;87:17-23.

8. Jiang SB, Pope C, Al Jarrah KM, Kung JH, Bortfeld T, Chen GT. An experimental investigation on intra-fractional organ motion effects in lung IMRT treatments. Phys Med Biol 2003;48:1773-84.

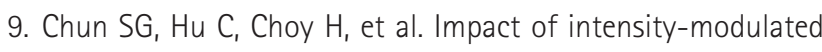
radiation therapy technique for locally advanced non-smallcell lung cancer: a secondary analysis of the NRG Oncology RTOG 0617 randomized clinical trial. J Clin Oncol 2017;35:5662.

10. Goldstraw P, Crowley J, Chansky K, et al. The IASLC Lung Cancer Staging Project: proposals for the revision of the TNM stage groupings in the forthcoming (seventh) edition of the TNM Classification of malignant tumours. J Thorac Oncol 2007:2:706-14.

11. National Comprehensive Cancer Network. NCCN Clinical Practice Guidelines in Oncology: non-small cell lung cancer [Internet]. Fort Washington, PA: National Comprehensive Cancer Network; c2018 [cited 2018 Aug 25]. Available from: https://www.nccn.org/professionals/physician_gls/default. aspx.

12. MacFarlane $M$, Hoover DA, Wong $E_{1}$ et al. Evaluation of unified intensity-modulated arc therapy for the radiotherapy of headand-neck cancer. Radiother Oncol 2016;119:331-6.

13. Jiang $X, L i T$, Liu $Y$, et al. Planning analysis for locally advanced lung cancer: dosimetric and efficiency comparisons between 
intensity-modulated radiotherapy (IMRT), single-arc/partialarc volumetric modulated arc therapy (SA/PA-VMAT). Radiat Oncol 2011;6:140.

14. Gagliardi G, Constine LS, Moiseenko V, et al. Radiation dosevolume effects in the heart. Int J Radiat Oncol Biol Phys 2010;76(3 Suppl):S77-85.

15. Schallenkamp JM, Miller RC, Brinkmann DH, Foote T, Garces YI. Incidence of radiation pneumonitis after thoracic irradiation: dose-volume correlates. Int J Radiat Oncol Biol Phys 2007;67:410-6.

16. Wang $S$, Liao $Z$, Wei $X$, et al. Analysis of clinical and dosimetric factors associated with treatment-related pneumonitis (TRP) in patients with non-small-cell lung cancer (NSCLC) treated with concurrent chemotherapy and three-dimensional conformal radiotherapy (3D-CRT). Int J Radiat Oncol Biol Phys 2006;66:1399-407.

17. Wu Z, Xie C, Hu M, et al. Dosimetric benefits of IMRT and VMAT in the treatment of middle thoracic esophageal cancer: is the conformal radiotherapy still an alternative option? J Appl Clin Med Phys 2014;15:93-101.

18. Chan OS, Lee MC, Hung AW, Chang AT, Yeung RM, Lee AW. The superiority of hybrid-volumetric arc therapy (VMAT) technique over double arcs VMAT and 3D-conformal technique in the treatment of locally advanced non-small cell lung cancer: a planning study. Radiother Oncol 2011;101:298-302.

19. Graham MV, Purdy JA, Emami B, et al. Clinical dose-volume histogram analysis for pneumonitis after 3D treatment for non-small cell lung cancer (NSCLC). Int J Radiat Oncol Biol Phys 1999:45:323-9.

20. Kwa SL, Lebesque JV, Theuws JC, et al. Radiation pneumonitis as a function of mean lung dose: an analysis of pooled data of 540 patients. Int J Radiat Oncol Biol Phys 1998;42:1-9.

21. Kristensen CA, Nottrup TJ, Berthelsen AK, et al. Pulmonary toxicity following IMRT after extrapleural pneumonectomy for malignant pleural mesothelioma. Radiother Oncol 2009;92:969.

22. Rosen II, Fischer TA, Antolak JA, et al. Correlation between lung fibrosis and radiation therapy dose after concurrent radiation therapy and chemotherapy for limited small cell lung cancer. Radiology 2001;221:614-22.

23. Khalil AA, Hoffmann L, Moeller DS, Farr KP, Knap MM. New dose constraint reduces radiation-induced fatal pneumonitis in locally advanced non-small cell lung cancer patients treated with intensity-modulated radiotherapy. Acta Oncol 2015;54:1343-9.

24. Belliere A, Girard N, Chapet O, et al. Feasibility of highdose three-dimensional radiation therapy in the treatment of localised non-small-cell lung cancer. Cancer Radiother 2009;13:298-304.

25. Gayed I, Gohar S, Liao Z, McAleer M, Bassett R, Yusuf SW. The clinical implications of myocardial perfusion abnormalities in patients with esophageal or lung cancer after chemoradiation therapy. Int J Cardiovasc Imaging 2009;25:487-95.

26. Schytte T, Hansen O, Stolberg-Rohr T, Brink C. Cardiac toxicity and radiation dose to the heart in definitive treated non-smal cell lung cancer. Acta Oncol 2010;49:1058-60.

27. Sasaoka M, Nishikawa A, Futami T, Nishida K, Miwa H, Kadoya K. Rectal dose reduction using three-dimensional conformal radiotherapy for locally advanced prostate cancer: a combination of conformal dynamic-arc and five-static field technique. Radiother Oncol 2009;90:318-24. 